\title{
LETTERS
}

\section{Recalling high-strength opioid formulations would cause harm}

As a family doctor and palliative care enhanced competency resident, I was frustrated to read Mr. Matthew Herder and Dr. David Juurlink's commentary recommending a ministerial recall of highstrength opioid formulations. ${ }^{1}$ The authors' well-intentioned suggestion, if acted on, would bring considerable harm to patients suffering with pain from incurable diseases, particularly advanced cancer.

Many of my patients have metastatic disease characterized by extensive bone disease and soft tissue invasion. They are able to maintain good quality of life and function at home, partly through the benefits provided by the very high-dose opioid formulations demonized in the article. Without them, these patients may suffer from debilitating pain and associated anxiety, depression and loss of function, which can lead to hospital admission and even earlier death.

The authors of the commentary cite a recent clinical practice guideline on chronic noncancer pain to support their recommendation. ${ }^{2}$ Some recommendations in this guideline, particularly those on dosing, which are based on lower-quality evidence, remain controversial. The guideline also specifically excludes cancer pain from its scope. Although we might wish cancer did not exist, 1 in 2 Canadians will develop cancer in their lifetimes, half of those will die from it, and many of them will have pain requiring opioid analgesia., ${ }^{3,4}$ Dismissing the pill burden in these patients as a "relatively minor inconvenience" trivializes the constant pall of medical care over their daily lives.

The comparison to thalidomide is pertinent because thalidomide is also a drug with utility in treating cancer, and it remains available for patients with multiple myeloma. That a drug can be harmful in some patients is not a reason to deny it to all patients - if that were the case, we'd have no pharmaceuticals at all.

A ministerial recall is an inappropriate sledgehammer that would have questionable benefit while undoubtedly causing harm to my patients. A more nuanced approach - educating clinicians and patients about the harms of high-dose opioids in chronic noncancer pain, tracking doses and optimizing nonopioid management - would offer greater benefit while minimizing the inadvertent harms of prohibition.

\section{Kevin Wade MD}

Palliative care resident, University of

British Columbia, Vancouver, BC

Cite as: CMAJ 2019 March 4;191:E265. doi: $10.1503 / \mathrm{cmaj} .71587$

\section{References}

1. Herder M, Juurlink D. High-strength opioid formulations: the case for a ministerial recall. CMAJ 2018;190:E1404-5.

2. Busse JW, Craigie S, Juurlink DN, et al. Guideline for opioid therapy and chronic noncancer pain. CMAJ 2017;189:E659-66.

3. Canadian Cancer Society. Cancer statistics at a glance. Available: www.cancer.ca/en/cancer -information/cancer-101/cancer-statistics-at-a -glance (accessed 2019 Jan. 31).

4. Teunissen SC, Wesker W, Kruitwagen CJ, et al. Symptom prevalence in patients with incurable cancer: a systematic review. J Pain Symptom Manage 2007;34:94-104.

Competing interests: None declared. 